\title{
Safety and outcomes of subconjunctival allogenic mesenchymal stem cell transplantation in canine experimental corneal defects
}

\author{
Ju-Won Kim ${ }^{1, *}$, So-Young Lee ${ }^{1}$, Hee-Myung Park ${ }^{1,2}$ \\ ${ }^{1}$ Department of Veterinary Internal Medicine, College of Veterinary Medicine, and \\ ${ }^{2}$ BK21 Basic \& Diagnostic Veterinary Specialist Program for Animal Diseases, Konkuk University, Seoul 143-701, Korea
}

(Received: January 3, 2012; Revised: June 8, 2012; Accepted: July 2, 2012)

\begin{abstract}
Corneal injury is very common clinical condition in veterinary medicine and delayed or incomplete corneal healing has the potential of vision loss due to the loss of corneal transparency. For the reconstruction of corneal epithelium, tissue graft and cell transplantation have been prosperously investigated. The purpose of this study was to evaluate the clinical value and short-term safety of application of cultured allogenic mesenchymal stem cells (MSCs) in the treatment of canine experimental corneal defect. Corneal defects were surgically generated in the central corneas of healthy beagle dogs and cultured canine allogenic MSCs were transplanted via subconjunctival injection. Although mean healing time, the rate of epithelial regeneration, and the degree of corneal transparency were not significantly improved after MSC transplantation, significant immune reaction or incompatibility reaction was not detected except transient local irritation. These results propose the possibility of MSC application as a new regenerative medicine in canine ocular disorders.
\end{abstract}

Keywords : cornea, dogs, mesenchymal stem cell, transparency

\section{Introduction}

The cornea is composed of three compartments, the outer epithelium, middle stroma, and inner endothelium, and the outer epithelium are vulnerable to various type of injury [5]. Healing of the corneal epithelium proceeds through cell migration, proliferation, and remodeling, which resemble other epithelial healing processes [24], and epithelial cells of corneo-scleral junction called limbal epithelial stem cells play a major role in corneal reconstruction $[8,20]$. But damage of the limbal area can result to delayed or non-healing corneal defect, which condition is called as limbal stem cell deficiency $[12,19,20]$. In cases of non-healing corneal wounds, conjunctival graft, amniotic membrane transplantation, lamellar keratoplasty, and limbal stem cell transplantation have been used to reconstruct corneal defect $[6,8,13]$. In regenerative medicine, many previous studies showed that adult mesenchymal stem cells (MSCs) could be a cellular source for various injured tissue. In corneal injury, MSCs have been investigated as an alternative to limbal epithelial stem cells because of the profusion of cell source and the relative convenience of cell collection and ex vivo expansion [7, 13]. Moreover, some previous studies showed the immunosuppressive activity of MSCs, which might allow allogenic and xenogenic MSC transplantation not using immunosup- pressive treatment $[15,17]$. The purpose of this study is to investigate whether local transplantation of MSCs could affect the corneal healing process in dogs. At that same time, the short-term safety of subconjunctival transplantation of allogenic MSCs is investigated by histological comparisons of the MSC-treated corneas and the vehicle-treated corneas.

\section{Materials and Methods}

\section{Experimental animals}

All experimental protocols for this study were approved by the Institutional Animal Care and Use Committee of Konkuk University (KU IACUC KU08027), and all experimental procedures were carried out under the guidelines of the committee. Five adult female beagle dogs, aged 3 5 years and weighing $6.9 \sim 13.1 \mathrm{~kg}$, were used. The experimental dogs were selected through ocular examination including vision assessment, measurement of tear production and intraocular pressure, and confirmation of corneal integrity using Fluorescein staining. At the end of the experiments, all experimental animals were euthanized.

\section{Generation of experimental corneal wound}

Under general anesthesia using intravenous injection of tiletamine-zolazepam (10 mg/kg; Virbac, France) each eyes

*Corresponding author

Tel: +82-2-450-3664, Fax: +82-2-444-4396

E-mail: vetzoo@konkuk.ac.kr 


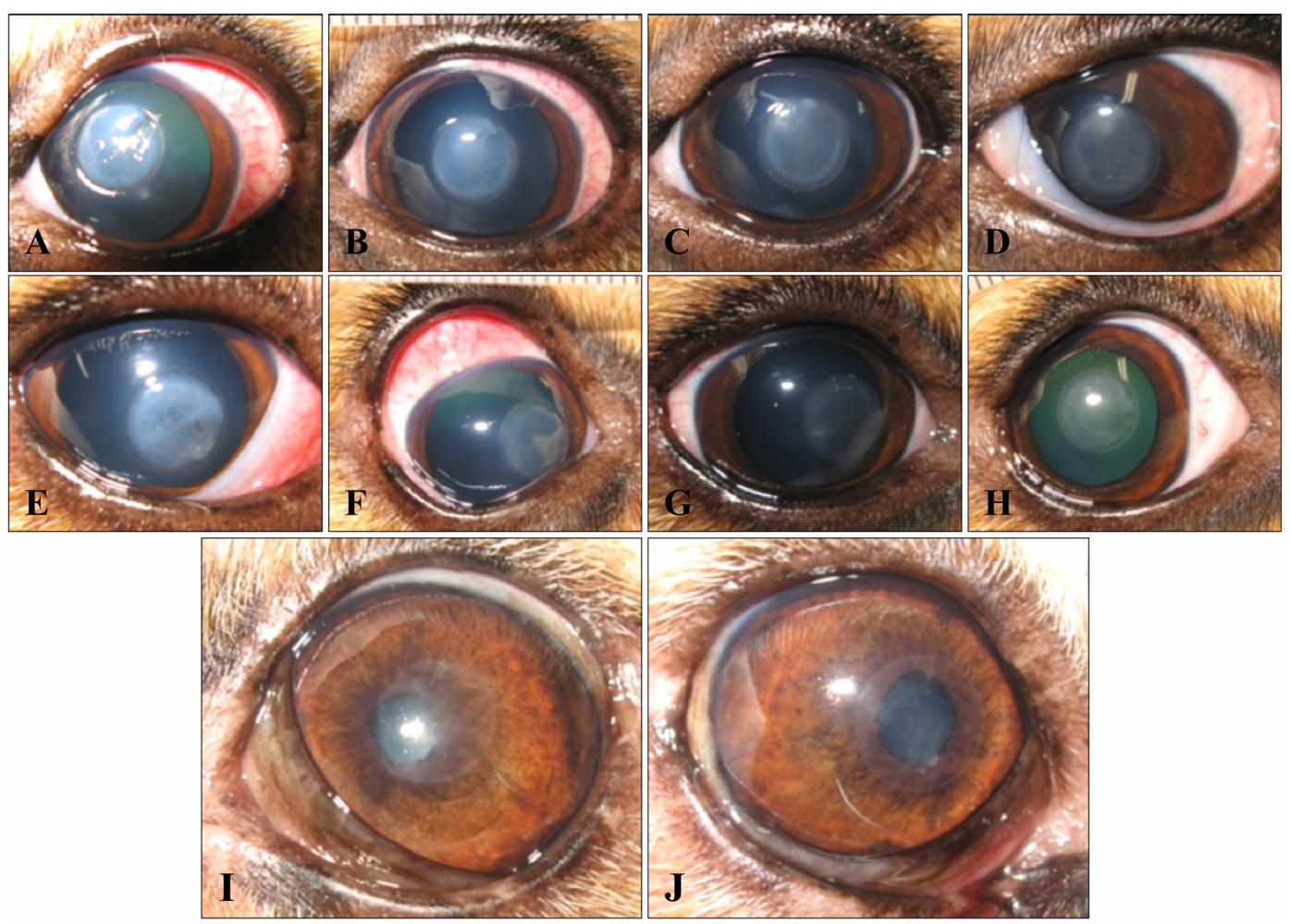

Fig. 1. Clinical outcomes of subconjunctival injection of mesenchymal stem cells (MSC) and phosphate buffered saline in experimental corneal defects on day 1 (A, E), 3 (B, F), 5 (C, G), 7 (D, H), and 24 (I, J). Control group (A-D) and MSC group (E-H) showed no significant difference in the healing process. At the early stage of healing, the MSC group revealed characteristic conjunctival hyperemia and focal subconjunctival swelling $(\mathrm{F})$, but these signs demonstrated variance among individuals and disappeared as time passed. Long-term clinical outcomes were evaluated on day 24, and the MSC group (J) showed more transparenency, and the area of the remained corneal opacity was smaller than in the control group (I). No conjunctival hyperemia or subconjunctival swelling was detected.

of the dogs were disinfected with $0.2 \%$ povidone-iodine solution (Sungkwang, Korea). For corneal trephination, topical $0.5 \%$ proparacaine hydrochloride (Alcon Korea, Korea) and eyelid speculum were applied and a 6-mm diameter biopsy punch was placed in the center of the cornea and pressed. The depth of the incision was set to approximate the anterior third of the cornea, the incised cornea was removed. Following the generation of a round corneal wound, fluorescein staining was applied to confirm the corneal defect without descemetocele (Fig. 1). This operation was performed on both eyes of all five dogs. After surgery, analgesics using tramadol $(5 \mathrm{mg} / \mathrm{kg}$; Yuhan Corporation, Korea) was administered. Topical gentamicin sulfate (Sam-il Pharm, Korea) eye drop was given three times a day for 7 days. The time of corneal ulcer generation was counted as day 0 .

\section{Subconjunctival MSC transplantation}

Canine allogenic MSCs were isolated from the bone marrow of healthy donor dogs and cultivated on. The procedure is described in our previous report [10]. Briefly, isolated canine bone marrow-derived MSCs were cultivated and passage of three to five MSCs were labeled with fluorescent dye for cell tracing using a carboxyfluorescein diacetate-succin- imidyl ester (CFDA-SE) cell tracer kit (Molecular Probes, USA) according to the manufacturer's instruction. The probecoated MSCs were collected and prepared to $2 \times 10^{6} \mathrm{MSCs}$ in $100 \mu \mathrm{L}$ phosphate buffered saline (PBS) for transplantation. Twenty-four hours after corneal wound generation, the dogs were sedated using medetomidine $(400 \mu \mathrm{g} / \mathrm{kg}, \mathrm{IM}$; Pfizer, USA). MSCs were transplanted into the right eye of each dog via subconjunctival injection using a $25 \mathrm{G}$ syringe. The same volume of PBS was injected into the left eye as the vehicle control. No experimental dogs received any immunesuppressive treatment.

\section{Clinical ocular examination}

Ocular examination of each wounded eye was performed daily until day 10 . Vision was assessed by menace reflex and Dazzle response, and the corneal surface and anterior chamber were examined under a hand-held light daily and photographed. The corneal epithelial defects were measured by fluorescein staining, and complete epithelial healing was determined when no fluorescein was retained on the corneal surface. The degree of the remaining corneal opacity and the size of the existing corneal epithelial defect were graded by a scoring system following the method of a previous study [12] 
Table 1. The clinical scoring system for corneal ulcer healing used in the experiment

\begin{tabular}{cll}
\hline \hline Score & \multicolumn{1}{c}{ Corneal transparency } & \multicolumn{1}{c}{ Size of corneal epithelial defect } \\
\hline 0 & Totally clear & No fluorescein uptake \\
1 & Trace or faint corneal haze & $<25 \%$ fluorescein stain of original wound \\
2 & Mild haze of minimal density & $25 \sim 50 \%$ fluorescein stain of original wound \\
3 & Moderately dense opacity partially obscuring inner ocular structure & $50 \sim 75 \%$ fluorescein stain of original wound \\
4 & Severely dense opacity completely obscuring inner ocular structure & $>75 \%$ fluorescein stain of original wound \\
\hline
\end{tabular}

with modifications (Table 1). Ophthalmic examination and clinical scoring were performed by two blinded investigators.

\section{Histological analysis of healed cornea}

On day 10, 3 of the experimental dogs were sacrificed, and both eyes were enucleated. The other 2 dogs were sacrificed on day 24 . The eyes were fixed in $10 \%$ neutral formalin and dissected along the center of the corneal defects, and then processed for histological examination. Sections of the MSCand PBS-treated eyes were stained with H\&E stain and then examined under a light microscope.

\section{Tracing of injected MSCs in the ocular tissue}

To determine the fate of transplanted MSCs, the unstained sections of the MSC group were examined under a fluorescence microscope (BK51; Olympus, Japan). Detected fluorescence positive cells were considered transplanted MSCs pre-labeled with CFDA-SE. The examination was repeated for the sections of the control group.

\section{Statistical analysis}

All data in this experiment were expressed as mean \pm standard deviation $(\mathrm{X} \pm \mathrm{SD})$. For statistical analyses, non-parametric tests were performed using the SPSS (ver 12.0; SPSS, USA). Comparisons of two mean values between groups were evaluated by the non-parametric Mann-Whitney U test. A probability value of $p<0.05$ was considered statistically significant.

\section{Results}

\section{Clinical evaluation of MSC transplantation in corneal defects}

All experimental dogs showed no systemic abnormality and maintained normal food consumption and activity during the entire experimental period. Mild ocular discharge and squinting were observed for a few days after corneal injury but there were no complications of corneal ulcer including stromal melting or perforation.

On examination of corneal reepithelialization using fluorescein dye, both groups showed gradual epithelial healing. All defected corneas showed negative fluorescein stain from day 3 to 7 , and the initial thick corneal opacity of ulcerated area became scattered. The mean healing time (MHT) was shorter in MSC group than in control group (4.8 \pm 0.98 day vs. $5.2 \pm 0.33$ day), but the difference was not significant
Table 2. Comparison of mean healing time and clinical score of MSC- and PBS-treated corneas

\begin{tabular}{lcc}
\hline \hline & $\begin{array}{c}\text { Control } \\
\text { group }\end{array}$ & $\begin{array}{c}\text { MSC } \\
\text { group }\end{array}$ \\
\hline Mean healing time $($ day) & $5.2 \pm 0.33$ & $4.8 \pm 0.98$ \\
Clinical score at day $3^{\dagger, 8}$ & $3.4 \pm 1.02$ & $3.2 \pm 1.17$ \\
Clinical score at day $7^{\dagger, *}$ & $1.4 \pm 0.49$ & $1.6 \pm 0.49$ \\
\hline
\end{tabular}

All results are mean $\pm \mathrm{SD}$. ${ }^{*}$ Mean healing time means the day of the negative fluorescein stain of the cornea. ${ }^{\star}$ A clinical score of 0 means complete corneal transparency and complete epithelial healing. ${ }^{\S}$ Day 3 is the time of the fastest complete reepithelialization in one experimental dog. "Day 7 is the time of complete healing of all experimental corneal ulcers. MSC: mesenchymal stem cells, PBS: phosphate buffered saline.

(Table 2). The mean clinical scores of the two groups on day 3 and 7 were also not significantly different (Table 2). But, distinctively, the upper bulbar conjunctiva of the injection area was more hyperemic in the MSC group, and focal subconjunctival swelling was observed although signs of ocular pain or severe ocular discharge were not examined (Fig. 1F). The degree of conjunctival hyperemia and subconjunctival swelling was variable depending on the individuals. These signs continued until approximately from day 7 to 9 and gradually disappeared. On day 10, only two eyes in MSC group showed focal subconjunctival swelling and hyperemia on the MSC injection site of the conjunctiva. The signs of the other three dogs had completely vanished. Although the MHT was not different between groups, the other two dogs were monitored for more than 2 weeks, and ocular examination was done by day 24 . On day 24 , all of the eyes showed no significant abnormality. Grossly, the corneas of MSC group showed more transparent that those of control group. The degree of the remaining corneal opacity was also higher in control group than in MSC group (Fig. 1).

\section{Histological comparison of healed corneas between groups}

On day 10, complete epithelial regeneration with hyperplastic reepithelialization in the ulcerated corneas was demonstrated in both groups. Arrangement of the corneal stroma was somewhat disturbed but the evidence of inflammation or corneal neovascularization was not examined (Figs. 2A and B). Subconjunctival space of the MSC group where abnormal focal swelling was detected demonstrated a focal area of 


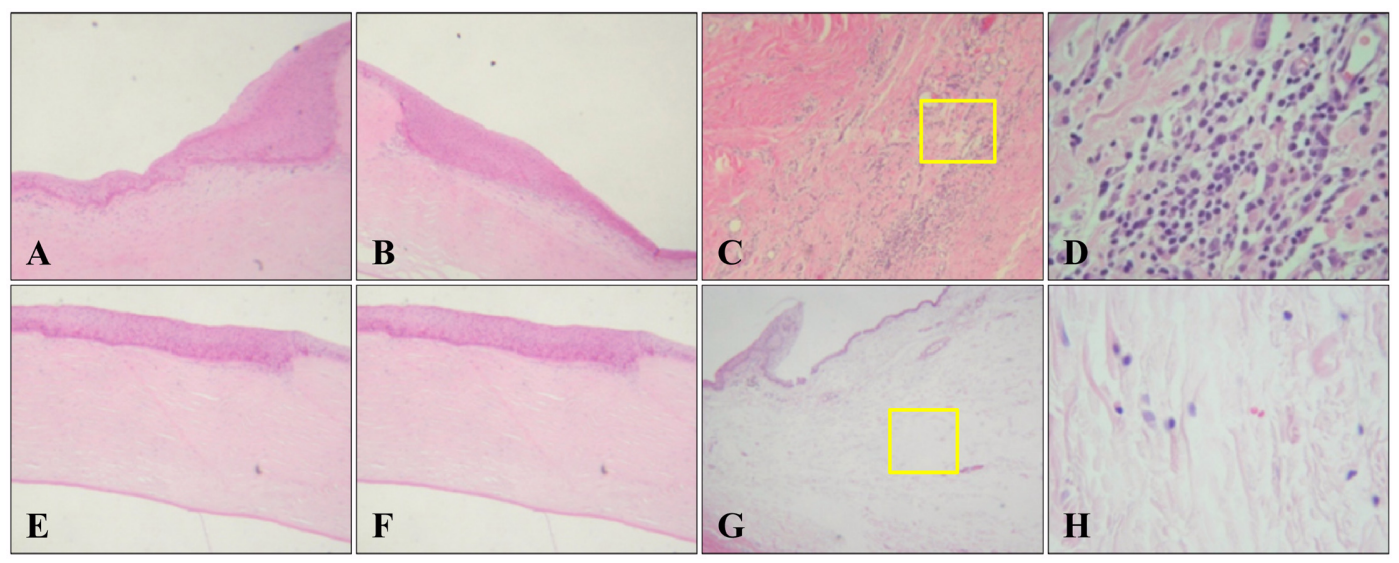

Fig. 2. Histological comparison of healed corneal defects on day 10 and 24 . The healed corneas on day 10 of the MSC group (A) and the control group (B) showed epithelial hyperplasia and increased keratocytes, but there was no significant. (C) Subconjunctival space of the MSC group on day 10 demonstrated varied degrees of increased cellularity. (D) Higher magnification of the area of increased cell density. (E) The healed corneas on day 24 of MSC group and control group (F). The thickness of the hyperplastic corneal epithelium became thinner compared to that of day 10. Loss of keratocytes was revealed, and those changes were identical in both groups. (G) Subconjunctival space of the MSC group on day 24. (H) higher magnification of the boxed area. Cellular infiltration was not detected, and there was no difference between the two groups. A, B, F: $\times 100$; C and G: $\times 200$; D and H: $\times 400$.
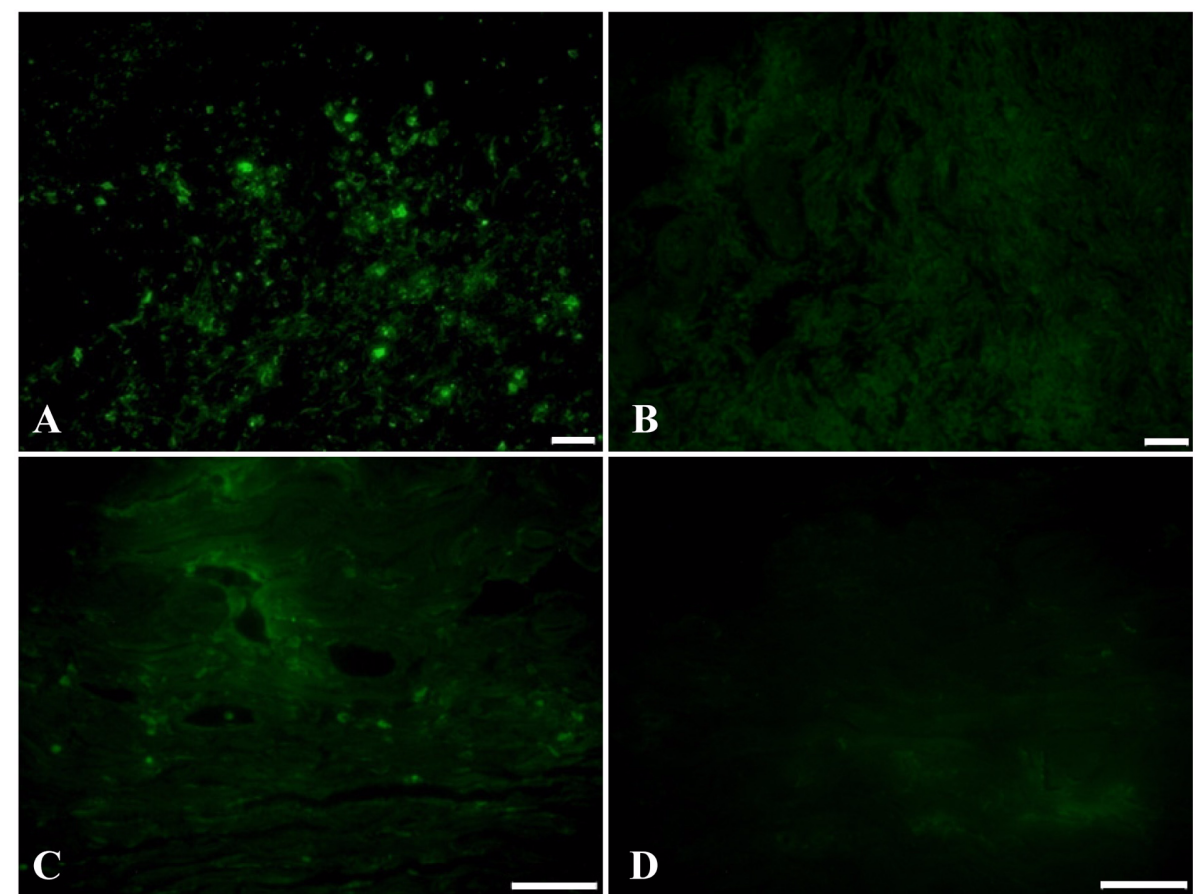

Fig. 3. Detection of transplanted MSCs in the ocular tissue. On day 10, a small number of CFDA-SE labeled cells were detected in the MSC group as fluorescent positive cell morphology only in the subconjunctival area (A). Fluorescent positive cells were not detected in the corneal area. The control group showed no positive signal (B). On day 24 , the positive cells had almost completely disappeared in the MSC group (C), and no cells were detected in the corneal epithelium and stroma, either. Sections of the control group also showed no positive cells both on day 10 and 24 (D). Scale bars $=50 \mu \mathrm{m}$.

variable increase in cellularity compared to other subconjunctival areas (Figs. 2C and D). This change was not observed in the control group. The degree of cellular infiltration varied among individual eyes. On day 24 , the hyperplastic regenerated corneal epithelium became thinner in each group, and the histological morphology was similar in both groups (Figs. $2 \mathrm{E}$ and $\mathrm{F}$ ). The increased cellular component of the MSC group disappeared, and the subconjunctival space of the groups showed no histological difference (Figs. 2G and H). 


\section{Engraftment of transplanted MSCs after SCI}

On day 10, fluorescent positive cells were detected in the MSC group in the area of the subconjunctival space. But the number of labeled cells was minimal, and the cells were not detected in the corneal epithelium or stroma. On day 24 , the remaining fluorescent positive cells were seldom detected in subconjunctival area and there were no fluorescent positive cells in corneal epithelium (Fig. 3).

\section{Discussion}

The cornea is the one of the surface organs prone to damage by various outer pathogens. However, investigations regarding MSC transplantation in wounded corneas are relatively limited compared to other organ damage. When the cornea is injured, many cases are healed without complications such as neovascularization and conjunctivalization [1]. But too much destruction of cornea could result in delayed or incomplete healing or loss of transparency, thereby requiring additional therapeutic strategy $[1,12]$. In veterinary practice, surgical repair of broad or deep corneal defects including corneal perforation and melting ulcer is accomplished by conjunctival graft or amniotic membrane and mucosal membrane transplantation $[6,21]$. But post-operative inflammation and resulting scar formation or corneal neovascularization are remained unsolved [22]. At this point, the regenerative ability of MSCs attracted notice, and a few investigators started to investigate MSC transplantation as an alternative source of bioengineering for reconstruction of damaged corneas.

In the present study, MSCs showed no significant positive effect in non-complicated surgical corneal wound healing. The rate of corneal reepithelialization and recovery of corneal transparency was not significantly different between MSC group and control group. A healthy cornea has excellent self-renewal activity, and in normal conditions, damaged corneal epithelium is healed by reepithelialization. A previous study suggested that wound healing in normal tissue might proceed at a maximal rate, therefore MSCs might not demonstrate significant effects on the preexisting optimal healing process [3]. The results of this experiment also suggested a similar proposal, and this assumption is supported by the previous results that systemically transplanted MSCs showed greater mobilization and engraft into wound sites in animals that had poorer tissue resident stem cells than healthy animals, and the promoting effects of MSCs were distinct in diabetic animals but not in normal animals $[2,3$, 14]. From the same point of view, this experiment revealed a considerable difference among individual experimental dogs; this might be due to the difference in the capacity of their own healing apparatus and tissue resident stem cells.

Meanwhile, this study showed the possibility of the subconjunctival allogenic MSC injection in the short run. Subconjunctival injection is an effective route of intraocular drug delivery, and several studies have used the subconjunctival route for transplantation of autologous blood cells, fibroblasts, and xenogenic neural progenitor cells without complications $[9,11]$. In this experiment, injection of MSCs into the subconjunctival space induced local stimulation such as conjunctival hyperemia and focal subconjunctival swelling in the early stage. Histological evidence of cellular infiltration was detected; thus, the potential risk of granuloma formation was not excluded. But, until day 10, the signs of local irritation were gradually disappeared and post-injectional granuloma did not observed. On cell tracing, fluorescent positive MSCs were rapidly disappeared and little detected on day 24 . As a result, the histological comparison of the MSC-injected conjunctiva and the PBS-injected conjunctiva showed no difference, and there were no signs of abnormal immune reaction and inflammatory infiltration. In contrast, previously the development of an immune response to transplanted allogenic MSCs in swine has been reported [18], and the occurrence of inflammatory pseudotumor post-hematopoietic stem cell transplantation has rarely been reported in human patients [4]. The present study demonstrated that subconjunctival injection of MSCs did not provoke severe systemic immune response and local glanuloma formation thus subconjunctival injection might be convenient and safer route for MSC transplantation than systemic injection in ocular diseases.

This study failed to demonstrate the engraftment of transplanted MSCs. On day 10, pre-labeled cells were sparsely detected in the conjunctival region, but fluorescent positive cells were not detected in the corneal epithelium, stroma, or endothelium. Moreover, as time passed, the detected cells were disappeared even in the conjunctival region. This result suggested that subconjunctivally injected MSCs did not migrate to the wounded cornea and removed by unknown mechanism. In contrast, other studies about MSC transplantation in the wounded cornea using direct application to the wounded corneal epithelium and systemic injection showed engraftment of transplanted MSCs in the cornea $[16,23]$. The difference may be due to the route of transplantation. But in the previous studies, the engrafted MSCs failed to transdifferentiate into corneal epithelial cells, and therapeutic effects of MSCs through paracrine effects of MSCs were suggested, which suppress inflammation and inhibit angiogenesis $[13,16]$. In this study, neither migration of transplanted MSCs nor differentiation was detected. Thus, the authors propose that subconjunctivally transplanted MSCs may contribute the corneal epithelial healing process via paracrine action although transplanted MSCs fail to migrate and were short-lived. The exact mechanism of action and the final fate of transplanted MSCs have still not been fully clarified. Further investigations are essential prior to the application of allogenic MSCs in practical cases.

\section{Acknowledgments}

The authors thank to Prof. Dong In Jung (Gyeongsang 
National University, Korea) for his encouragement and technical advice.

\section{References}

1. Azar DT. Corneal angiogenic privilege: angiogenic and antiangiogenic factors in corneal avascularity, vasculogenesis, and wound healing (an American Ophthalmological Society thesis). Trans Am Ophthalmol Soc 2006, 104, 264-302.

2. Badiavas EV, Falanga V. Treatment of chronic wounds with bone marrow-derived cells. Arch Dermatol 2003, 139, 510-516.

3. Badillo AT, Redden RA, Zhang L, Doolin EJ, Liechty KW. Treatment of diabetic wounds with fetal murine mesenchymal stromal cells enhances wound closure. Cell Tissue Res 2007, 329, 301-311.

4. Bahat G, Kalayoglu-Besisik S, Ozturk S, Kilicaslan I, Tunc M, Sargin D. Inflammatory pseudotumor following hematopoietic stem cell transplantation: a new case and review of the literature. Bone Marrow Transplant 2007, 40, 915-918.

5. Baldwin HC, Marshall J. Growth factors in corneal wound healing following refractive surgery: A review. Acta Ophthalmol Scand 2002, 80, 238-247.

6. Barros PS, Safatle AMV, Godoy CA, Souza MSB, Barros LFM, Brooks DE. Amniotic membrane transplantation for the reconstruction of the ocular surface in three cases. Vet Ophthalmol 2005, 8, 189-192.

7. Bartholomew A, Sturgeon C, Siatskas M, Ferrer K, McIntosh K, Patil S, Hardy W, Devine S, Ucker D, Deans R, Moseley A, Hoffman R. Mesenchymal stem cells suppress lymphocyte proliferation in vitro and prolong skin graft survival in vivo. Exp Hematol 2002, 30, 42-48.

8. Dua HS, Azuara-Blanco A. Limbal stem cells of the corneal epithelium. Surv Ophthalmol 2000, 44, 415-425.

9. Hwang JM, Kee C. Injection of cultured autologous fibroblasts into the subconjunctival space of rabbits treated with mitomycin C. Am J Ophthalmol 2006, 142, 259-263.

10. Jung DI, Ha J, Kim JW, Kang BT, Yoo JH, Park C, Lee JH, Park HM. Canine mesenchymal stem cells derived from bone marrow: isolation, characterization, multidifferentiation, and neurotrophic factor expression in vitro. J Vet Clin 2008, 25, 458-465.

11. Kuznetsov SL, Nikolaeva LR, Spivak IA, Chentsova EV, Poltavtseva RA, Marei MV, Sukhikh GT. Effect of transplantation of cultured human neural stem and progenitor cells on regeneration of the cornea after chemical burn. Bull Exp Biol Med 2006, 141, 129-132.

12. Luengo Gimeno F, Lavigne V, Gatto S, Croxatto JO, Correa L, Gallo JE. Advances in corneal stem-cell transplantation in rabbits with severe ocular alkali burns. J Cataract Refract Surg 2007, 33, 1958-1965.
13. Ma Y, Xu Y, Xiao Z, Yang W, Zhang C, Song E, Du Y, Li L. Reconstruction of chemically burned rat corneal surface by bone marrow-derived human mesenchymal stem cells. Stem Cells 2006, 24, 315-321.

14. Mathews V, Hanson PT, Ford E, Fujita J, Polonsky KS, Graubert TA. Recruitment of bone marrow-derived endothelial cells to sites of pancreatic beta-cell injury. Diabetes 2004, 53, 91-98.

15. McFarlin K, Gao X, Liu YB, Dulchavsky DS, Kwon D, Arbab AS, Bansal M, Li Y, Chopp M, Dulchavsky SA, Gautam SC. Bone marrow-derived mesenchymal stromal cells accelerate wound healing in the rat. Wound Repair Regen 2006, 14, 471-478.

16. Oh JY, Kim MK, Shin MS, Lee HJ, Ko JH, Wee WR, Lee JH. The anti-inflammatory and anti-angiogenic role of mesenchymal stem cells in corneal wound healing following chemical injury. Stem Cells 2008, 26, 1047-1055.

17. Perin EC, Silva GV, Assad JAR, Vela D, Buja LM, Sousa ALS, Litovsky S, Lin J, Vaughn WK, Coulter S, Fernandes MR, Willerson JT. Comparison of intracoronary and transendocardial delivery of allogeneic mesenchymal cells in a canine model of acute myocardial infarction. J Mol Cell Cardiol 2008, 44, 486-495.

18. Poncelet AJ, Vercruysse J, Saliez A, Gianello P. Although pig allogeneic mesenchymal stem cells are not immunogenic in vitro, intracardiac injection elicits an immune response in vivo. Transplantation 2007, 83, 783-790.

19. Qu L, Yang X, Wang X, Zhao M, Mi S, Dou Z, Wang H. Reconstruction of corneal epithelium with cryopreserved corneal limbal stem cells in a rabbit model. Vet J 2009, 179, 392-400.

20. Revoltella RP, Papini S, Rosellini A, Michelini M. Epithelial stem cells of the eye surface. Cell Prolif 2007, 40, 445-461.

21. Vanore M, Chahory S, Payen G, Clerc B. Surgical repair of deep melting ulcers with porcine small intestinal submucosa (SIS) graft in dogs and cats. Vet Ophthalmol 2007, 10, 93-99.

22. Wichayacoop $\mathbf{T}$, Briksawan $\mathbf{P}$, Tuntivanich $\mathbf{P}$, YibchokAnun S. Anti-inflammatory effects of topical supernatant from human amniotic membrane cell culture on canine deep corneal ulcer after human amniotic membrane transplantation. Vet Ophthalmol 2009, 12, 28-35.

23. Ye J, Yao K, Kim JC. Mesenchymal stem cell transplantation in a rabbit corneal alkali burn model: engraftment and involvement in wound healing. Eye (Lond) 2006, 20, 482-490.

24. Zieske JD, Takahashi H, Hutcheon AEK, Dalbone AC. Activation of epidermal growth factor receptor during corneal epithelial migration. Invest Ophthalmol Vis Sci 2000, 41, 1346-1355. 